VOL. $71(2005) \quad[121-126]$

\title{
CONJUGAISON GÉODÉSIQUE EN RANG 1
}

\author{
HAMID-REZA FANAÏ
}

Soit $\left(M, g_{0}\right)$ une variété riemannienne compacte de courbure sectionnelle négative. Soit $g_{1}$ une autre métrique riemannienne sur $M$ de rang 1 . On montre que l'égalité des spectres marqués des longueurs de $g_{0}$ et $g_{1}$ implique que le flot géodésique de $g_{0}$ est un facteur de celui de $g_{1}$.

\section{INTRODUCTION}

Soit $(M, g)$ une variété riemannienne compacte. Il est bien connu qu'on peut représenter chaque classe de conjugaison $\langle\gamma\rangle$ de $\Gamma$ le groupe fondamental de $M$ par une classe d'homotopie libre de courbes dans $M$. On note $\ell_{g}(\gamma)$ la longueur d'une courbe de longueur minimale dans cette classe. Une telle courbe est toujours une géodésique périodique (qui peut être réduite à un point) et lorsque la métrique $g$ est sans points conjugués, alors toutes les géodésiques périodiques dans cette classe ont même longueur $\ell_{g}(\gamma)$. Si $g$ est de courbure négative, une telle géodésique périodique est unique.

Soit $\mathcal{C}$ l'ensemble des classes de conjugaison du groupe fondamental de $M$. Par définition, le spectre marqué des longueurs de la métrique $g$ est l'élément $\left(\ell_{g}(\gamma)\right)_{\gamma \in \mathcal{C}} \mathrm{du}$ produit direct $\mathbb{R}^{c}$. Il est important de savoir si ce spectre détermine la métrique. On aimerait surtout avoir des informations dynamiques, par exemple sur $\varphi_{t}^{g}$ le flot géodésique de $(M, g)$ défini sur $S_{g} M$ le fibré unitaire tangent. Notons que s'il y a une conjugaison entre les flots géodésiques de deux métriques, alors elles ont même spectre. Inversement, en courbure négative, un résultat de Hamenstädt ([4]) dit que l'égalité des spectres marqués implique l'existence d'une conjugaison géodésique de classe $C^{0}$.

Dans cette note, nous obtenons une version plus générale de ce résultat dans le cadre des variétés riemanniennes compactes de rang 1 , c'est à dire que la courbure est nonpositive mais il y a une géodésique hyperbolique, c'est à dire, une géodésique qui n'admet pas de champ de Jacobi parallèle perpendiculaire. Nous donnons une estimation valable en courbure négative ([7]) pour le cadre plus général des variétés de rang 1 . Nous utilisons la notion d'intersection des métriques ([7]) et également les résultats de [8] concernant la famille de mesures de Patterson-Sullivan en rang 1. Cela nous permettra de montrer le résultat principal de ce texte (comparer avec $[5,10]$ ).

Received 8th September, 2004

This research was in part supported by a grant from IPM (No. 83530035)

Copyright Clearance Centre, Inc. Serial-fee code: 0004-9727/05 \$A2.00+0.00. 
ThÉoRÈme 1.1. Soit $\left(M, g_{0}\right)$ une variété reimannienne compacte de courbure sectionnelle négative. Soit $g_{1}$ une autre métrique riemannienne sur $M$ de rang 1 . Si le spectre marqué des longueurs de $\left(M, g_{0}\right)$ est égal à celui de $\left(M, g_{1}\right)$, alors le flot géodésique de $\left(M, g_{0}\right)$ est un facteur de celui de $\left(M, g_{1}\right)$, c'est à dire, il existe une application continue et surjective $F: S_{g_{1}} M \rightarrow S_{g_{0}} M$ vérifiant $F \circ \varphi_{t}^{g_{1}}=\varphi_{t}^{g_{0}} \circ F$.

À la fin, nous donnons également quelques applications de ce théorème.

\section{PrÉliminaires}

Nous présentons tout d'abord quelques notions utiles.

ENTROPIE TOPOLOGIQUE. Soit $(M, g)$ une variété riemannienne compacte de rang 1. Pour tout réel $T>0$, on note $N_{g}(T)$ le nombre des géodésiques périodiques de période $\leqslant T$ (à classe d'homotopie libre près). Il est connu que ([6]) $h_{\text {top }}(g)$, l'entropie topologique du flot géodésique de $(M, g)$ est égale à

$$
h_{\text {top }}(g)=\lim _{T \rightarrow+\infty} \frac{\log N_{g}(T)}{T} .
$$

Si $g^{\prime}$ est une autre métrique riemannienne sur $M$ de rang 1 ayant même spectre marqué des longueurs que $g$, alors l'on a $h_{\text {top }}\left(g^{\prime}\right)=h_{\text {top }}(g)$.

INTERSECTION. Soit $(M, g)$ une variété riemannienne compacte. Rappelons la définition de l'intersection ([7]): soit $g^{\prime}$ une autre métrique riemannienne sur $M$. Pour toute mesure de probabilité $\mu$ sur $S_{g} M$ invariante par $\varphi_{t}^{g}$ le flot géodésique de $(M, g)$, l'intersection de $g^{\prime}$ par rapport à $g$ et $\mu$ est égale à:

$$
I_{\mu}\left(g, g^{\prime}\right)=\inf _{t>0} \frac{1}{t} \int_{S_{g} M} a(v, t) d \mu(v)
$$

où $a(v, t)=d_{g^{\prime}}\left(\widetilde{C}_{v}(0), \widetilde{C}_{v}(t)\right)$ et pour tout vecteur $v \in S_{g} M, \widetilde{C}_{v}(t)$ désigne le relevé de la géodésique paramétrée par la longueur d'arc, définie par $v$, dans $\widetilde{M}$ le revêtement universel de $M$.

Soient $\gamma$ et $\gamma^{\prime}$ deux géodésiques périodiques de $g$ et $g^{\prime}$ respectivement, donnant les longueurs minimales dans une même classe d'homotopie libre. Soit $\delta$ la mesure de Dirac normalisée, associée à $\gamma$ sur $S_{g} M$ invariante par le flot géodésique de $(M, g)$. Soit $\ell$ la $g$-longueur de $\gamma$ et $\ell^{\prime}$ la $g^{\prime}$-longueur de $\gamma^{\prime}$. Supposons $\ell=\ell^{\prime}$. Exactement comme dans [1], lorsque $g$ et $g^{\prime}$ n'ont pas de points conjugués, nous calculons $I_{\delta}\left(g, g^{\prime}\right)$, l'intersection de $g^{\prime}$ par rapport à $g$ et $\delta$ et obtenons:

$$
I_{\delta}\left(g, g^{\prime}\right)=\frac{\ell^{\prime}}{\ell}=1
$$

Par conséquent, si $g$ et $g^{\prime}$ ont même spectre marqué des longueurs, alors l'intersection de $g^{\prime}$ par rapport à $g$ et toute mesure de Dirac normalisée est égale à 1 . 
UNE ESTIMATION. Maintenant, supposons que $\left(M, g_{0}\right)$ est de courbure négative et que $g_{1}$ est une autre métrique riemannienne de rang 1 sur $M$ avec le même spectre marqué des longueurs que $g_{0}$. Soit $\mu_{\mathrm{BM}}$ la mesure de Bowen-Margulis de $g_{0}$. Il est bien connu que cette mesure est une limite des mesures de Dirac normalisées supportées sur des géodésiques périodiques. Le fait que l'intersection de $g_{1}$ par rapport à $g_{0}$ et toute mesure de Dirac normalisée est égale à 1 , avec des arguments similaires à ceux utilisés dans [1] ou $[5]$, nous donne $I_{\mu_{\mathrm{BM}}}\left(g_{0}, g_{1}\right)=1$. Il est à noter que l'on peut utiliser également la continuité de l'intersection par rapport à la mesure (en courbure négative par exemple, voir [2]).

\section{Preuve}

Dans [7] lorsque $g_{0}$ et $g_{1}$ sont toutes les deux de courbure négative et ont même entropie, il est démontré que $I_{\mu_{\mathrm{BM}}}\left(g_{0}, g_{1}\right) \geqslant 1$ avec égalité si et seulement si les flots géodésiques de $g_{0}$ et $g_{1}$ sont $C^{0}$-conjugués. Le théorème 1.1 est prouvé dès que l'on montre le lemme suivant.

LEMME 3.1. Soit $\left(M, g_{0}\right)$ une variété reimannienne compacte de courbure négative. Soit $g_{1}$ une autre métrique riemannienne sur $M$ avec la même entropie topologique que $g_{0}$. Soit $\mu_{\mathrm{BM}}$ la mesure de Bowen-Margulis de $g_{0}$. Alors $I_{\mu_{\mathrm{BM}}}\left(g_{0}, g_{1}\right) \geqslant 1$. Si $g_{1}$ est de rang 1 et il y a l'égalité, alors il existe une application continue et surjective $F: S_{g_{1}} M \rightarrow S_{g_{0}} M$ vérifiant $F \circ \varphi_{t}^{g_{1}}=\varphi_{t}^{g^{0}} \circ F$.

Prouvons ce lemme. En ce qui concerne l'inégalité, ceci est démontré dans [7]. Pour le cas d'égalité, il faut utiliser les arguments de [7] qui ont été adaptés pour des variétés de rang 1 dans [8]. L'idée principale est de construire une famille de mesures de PattersonSullivan $\left\{\mu_{p}\right\}_{p \in \widetilde{M}}$ sur $\widetilde{M}(\infty)$ le bord à l'infini tt de prouver une propriété locale essentielle de cette famille. Dans le cas d'égalité, il faut constater que ceci implique l'équivalence des mesures de Patterson-Sullivan associées. Une telle équivalence implique l'existence d'une conjugaison géodésique de classe $C^{0}$ en courbure négative ([7]). Seulement en rang 1 , on ne peut pas espérer que la conjugaison reste injective car entre deux points du bord, il peut y avoir plusieures géodésiques reliant ces deux points. C'est pour cela qu'on obtient un facteur de $\varphi_{t}^{g_{1}}$. L'autre point important est la comparaison des bords à l'infini pour les deux métriques. Par compacité de $M$, on sait que les deux espaces $\left(\widetilde{M}, \widetilde{g}_{0}\right)$ et $\left(\widetilde{M}, \tilde{g}_{1}\right)$ sont quasi-isométriques. D'autre part, la métrique $g_{0}$ étant de courbure négative, il est bien connu que $\Gamma$ est un espace hyperbolique au sens de Gromov et puisque cet espace est quasi-isométrique à $\left(\widetilde{M}, \widetilde{g}_{0}\right)$, ce dernier est donc un espace hyperbolique au sens de Gromov ainsi que $\left(\widetilde{M}, \widetilde{g}_{1}\right)$. Ceci implique ([3]) l'existence d'un homéomorphisme $P:\left(\widetilde{M}, \tilde{g}_{1}\right)(\infty) \rightarrow\left(\widetilde{M}, \tilde{g}_{0}\right)(\infty)$ qui est $\Gamma$ équivariante. En fait, tout rayon géodésique de $\left(\widetilde{M}, \tilde{g}_{1}\right)$ étant un quasi-rayon géodésique de $\left(\widetilde{M}, \widetilde{g}_{0}\right)$ est à distance de Hausdorff bornée d'un vrai rayon géodésique de $\left(\widetilde{M}, \widetilde{g}_{0}\right)$ et vice-versa. 
Maintenant, expliquons comment on a l'équivalence entre les mesures de PattersonSullivan de $g_{0}$ et $g_{1}$ lorsque $I_{\mu_{\mathrm{BM}}}\left(g_{0}, g_{1}\right)=1$. Pour simplifier, supposons que $h_{\mathrm{top}}\left(g_{0}\right)$ $=h_{\mathrm{top}}\left(g_{1}\right)=1$. Comme dans [7], pour tout $p \in \widetilde{M}$, il existe une constante $L$ telle que pour $\mu_{p}^{g_{0}}$-presque tout $\xi \in\left(\widetilde{M}, \tilde{g}_{0}\right)(\infty)$, il existe une suite $t_{n} \rightarrow \infty$ telle que si $y_{n}=\pi \varphi_{t_{n}}^{g_{0}} v_{p}(\xi)$ (où $\pi$ est la projection naturelle et $v_{p}(\xi)$ est le vecteur tangent à $p$ pointant vers $\xi$ ), alors

$$
\left|d_{g_{1}}\left(p, y_{n}\right)-I_{\mu_{\mathrm{BM}}}\left(g_{0}, g_{1}\right) t_{n}\right| \leqslant L
$$

D'où $\left|d_{g_{1}}\left(p, y_{n}\right)-t_{n}\right| \leqslant L$. Ceci avec la propriété locale des mesures de Patterson-Sullivan ([8]) donne pour $R>0$ constante:

$$
\frac{1}{c_{1}} e^{-d_{g_{1}}\left(p, y_{n}\right)} \leqslant \mu_{p}^{g_{1}}\left(\operatorname{pr}_{p}^{g_{1}}\left(B\left(y_{n}, R\right)\right)\right) \leqslant c_{1} e^{-d_{g_{1}}\left(p, y_{n}\right)}
$$

où $B(y, R)$ est la boule centrée en $y$ de rayon $R$ et $\operatorname{pr}_{p}$ désigne la projection sur le bord à l'infini le long des géodésiques. Ceci implique

$$
\frac{1}{c_{2}} e^{-d_{g_{0}}\left(p, y_{n}\right)} \leqslant \mu_{p}^{g_{1}}\left(\operatorname{pr}_{p}^{g_{1}}\left(B\left(y_{n}, R\right)\right)\right) \leqslant c_{2} e^{-d_{g_{0}}\left(p, y_{n}\right)}
$$

Si $R$ est plus grande que la distance de Hausdorff maximale $r$ des rayons géodésiques associés pour $g_{0}$ et $g_{1}$, on a

$$
\operatorname{pr}_{p}^{g_{0}}\left(B\left(y_{n}, R-r\right)\right) \subset \operatorname{pr}_{p}^{g_{1}}\left(B\left(y_{n}, R\right)\right) \subset \operatorname{pr}_{p}^{g_{0}}\left(B\left(y_{n}, R+r\right)\right)
$$

et on obtient

$$
\mu_{p}^{g_{0}}\left(\operatorname{pr}_{p}^{g_{1}}\left(B\left(y_{n}, R\right)\right)\right) \leqslant \mu_{p}^{g_{0}}\left(\operatorname{pr}_{p}^{g_{0}}\left(B\left(y_{n}, R+r\right)\right)\right) \leqslant c_{3} e^{-d_{g_{0}}\left(p, y_{n}\right)}
$$

et aussi

$$
c_{4} e^{-d_{g_{0}}\left(p, y_{n}\right)} \leqslant \mu_{p}^{g_{0}}\left(\operatorname{pr}_{p}^{g_{0}}\left(B\left(y_{n}, R-r\right)\right)\right) \leqslant \mu_{p}^{g_{0}}\left(\operatorname{pr}_{p}^{g_{1}}\left(B\left(y_{n}, R\right)\right)\right)
$$

On a alors

$$
\frac{1}{c_{5}} \leqslant \frac{\mu_{p}^{g_{0}}\left(\operatorname{pr}_{p}^{g_{1}}\left(B\left(y_{n}, R\right)\right)\right)}{\mu_{p}^{g_{1}}\left(\operatorname{pr}_{p}^{g_{1}}\left(B\left(y_{n}, R\right)\right)\right)} \leqslant c_{5}
$$

c'est à dire que $\left(d \mu_{p}^{g_{0}}\right) /\left(d \mu_{p}^{g_{1}}\right)(\xi)$ existe. À l'aide de ceci, comme dans [7], nous pouvons définir l'application $F$ de la manière suivante. Soit $v \in S_{g_{1}} \widetilde{M}$ et posons $\xi=\varphi_{+\infty}^{g_{1}}(v)$ et $\eta=\varphi_{-\infty}^{g_{1}}(v)$. On sait qu'il existe une unique géodésique de $\left(\widetilde{M}, \widetilde{g}_{0}\right)$ reliant $\eta$ à $\xi$. Il y a un unique $w \in S_{g_{0}} \widetilde{M}$ tangent à cette géodésique tel que $\left(d \mu_{\pi w}^{g_{0}}\right) /\left(d \mu_{\pi v}^{g_{1}}\right)(\xi)=1$. L'application $F: S_{g_{1}} \widetilde{M} \rightarrow S_{g_{0}} \widetilde{M}$ définie par $F(v)=w$ est la bonne puisqu'elle est $\Gamma$ invariante.

\section{Applications}

On a un facteur seulement, car $F$ n'est pas injective, mais si l'on considère la partie régulière du fibré, alors sur cette partie, $F$ est injective. Rappelons que la partie régulière du fibré unitaire tangent est tous les vecteurs tángents à des géodésiques hyperboliques. Il est connu que cette partie est un ouvert dense dans le fibré unitaire tangent. On a donc 
COROLla IRE 4.1. Soit $\left(M, g_{0}\right)$ une variété reimannienne compacte de courbure sectionnelle négative. Soit $g_{1}$ une autre métrique riemannienne sur $M$ de rang 1 . Si le spectre marqué des longueurs de $\left(M, g_{0}\right)$ est égal à celui de $\left(M, g_{1}\right)$, alors il y a une conjugaison géodésique entre un ouvert dense de $S_{g_{1}} M$ sur son image dans $S_{g_{0}} M$.

Il est intéressant de constater qu'avec les notations du lemme 3.1, si les entropies topologiques de $g_{0}$ et $g_{1}$ sont égales et qu'il y a une inégalité entre les spectres marqués des longueurs $\operatorname{SML}\left(g_{1}\right) \leqslant \operatorname{SML}\left(g_{0}\right)$, c'est à dire que pour toute classe de conjugaison $\langle\gamma\rangle$ du groupe fondamental de $M$, on a $\ell_{g_{1}}(\gamma) \leqslant \ell_{g_{0}}(\gamma)$, alors la même démarche montre que $I_{\mu_{\mathrm{BM}}}\left(g_{0}, g_{1}\right) \leqslant 1$ et on est donc dans le cas d'égalité du lemme.

COROLla IRE 4.2. Soit $\left(M, g_{0}\right)$ une variété reimannienne compacte de courbure sectionnelle négative. Soit $g_{1}$ une autre métrique riemannienne de rang 1 sur $M$ avec la même entropie topologique que $g_{0}$. Si SML $\left(g_{1}\right) \leqslant \operatorname{SML}\left(g_{0}\right)$, alors le flot géodésique de $g_{0}$ est un facteur de celui de $g_{1}$. En particulier, on a $\operatorname{SML}\left(g_{1}\right)=\operatorname{SML}\left(g_{0}\right)$.

Il est naturel de considérer également l'inégalité $\operatorname{SML}\left(g_{0}\right) \leqslant \operatorname{SML}\left(g_{1}\right)$. Pour ceci, nous pouvons obtenir un corollaire similaire. En effet, d'après les résultats de [9], on sait que la mesure d'entropie maximale $\mu_{\max }$ pour la métrique $g_{1}$ est unique et qu'elle est une limite des combinaisons des mesures de Dirac. Dans ce cas, avec l'inégalité supposée, on obtient $I_{\mu_{\max }}\left(g_{1}, g_{0}\right) \leqslant 1$. Maintenant le Lemme 3.1 reste vrai si l'on travaille avec $I_{\mu_{\max }}\left(g_{1}, g_{0}\right)$ car tous les arguments restent valables. On a donc

COROLla IRE 4.3. Soit $\left(M, g_{0}\right)$ une variété reimannienne compacte de courbure sectionnelle négative. Soit $g_{1}$ une autre métrique riemannienne de rang 1 sur $M$ avec la même entropie topologique que $g_{0}$. Si SML $\left(g_{0}\right) \leqslant \operatorname{SML}\left(g_{1}\right)$, alors le flot géodésique de $g_{0}$ est un facteur de celui de $g_{1}$. En particulier, on a $\operatorname{SML}\left(g_{0}\right)=\operatorname{SML}\left(g_{1}\right)$.

\section{REFERENCES}

[1] H.-R. Fanaï, 'Spectre marqué des longueurs et métriques conformément équivalentes', Bull. Belg. Math. Soc. 5 (1998), 525 528.

[2] A. Fathi et L. Flaminio, 'Infinitesimal conjugacies and Weil-Petersson metric', Ann. Inst. Fourier 43 (1993), 279-299.

[3] E. Ghys et P. de la Harpe, Sur les groupes hyperboliques d'après Mikhael Gromov, Progress in Mathematics 83 (Birkhauser Boston, Inc., Boston, MA, 1990).

[4] U. Hamenstädt, 'Time preserving conjugacies of geodesic flows', Ergodic Theory Dynamical Systems 12 (1992), 67-74.

[5] I. Kim, 'Ergodic theory and rigidity on the symmetric space of non-compact type', Ergodic Theory Dynamical Systems 21 (2001), 93-114.

[6] G. Knieper, 'Das Wachstum der Äquivalenzklassen geschlossener Geodätischer in kompakten Riemannschen Mannigfaltigkeiten', Arch. Math. (Basel) 40 (1983), 559-568.

[7] G. Knieper, 'Volume growth, entropy and the geodesic stretch', Math. Res. Lett. 2 (1995), 39-58. 
[8] G. Knieper, 'On the asymptotic geometry of nonpositively curved manifolds', Geom. Funct. Anal. 7 (1997), 755-782.

[9] G. Knieper, 'The uniqueness of the measure of maximal entropy for geodesic flows on rank 1 manifolds', Ann. Math. 148 (1998), 291-314.

[10] C.B. Yue, 'The ergodic theory of discrete isometry. groups on manifolds of variable negative curvature', Trans. Amer. Math. Soc. 348 (1996), 4965-5005.

Department of Mathematical Sciences

Sharif University of Technology

P.O.Box 11365-9415

Tehran

Iran
Institute for Studies in Theoretical

Physics and Mathematics (IPM)

\section{Tehran}

Iran

e-mail: fanai@sharif.ac.ir 